\title{
Experimental investigation of surface deformations at the nematic-isotropic interface : a new method to measure the Nehring-Saupe elastic constant $K_{13}\left(^{+}\right)$
}

\author{
S. Faetti $\left({ }^{*}\right)$ and V. Palleschi \\ Dipartimento di Fisica dell' Universitá di Pisa \\ and Gruppo Nazionale di Struttura della Materia del CNR (*), Piazza Torricelli 2, 56100 Pisa, Italy
}

(Reçu le 16 août 1984, accepté le 26 octobre 1984)

\begin{abstract}
Résumé. - Les déformations structurelles de l'interface nématique-isotrope d'un cristal liquide nématique dans un champ magnétique ont été étudiées théoriquement par de Gennes. Dans cet article nous généralisons la théorie de de Gennes en prenant en considération l'anisotropie des constantes élastiques, l'énergie d'ancrage finie et l'effet de la constante élastique $K_{13}$ de Nehring et Saupe. Nous trouvons une expression analytique simple qui relie l'angle maximum d'inclinaison de l'interface à l'angle polaire du directeur en surface, aux constantes élastiques, à la tension superficielle et au champ magnétique. Nous montrons que la mesure de cette inclinaison peut permettre d'obtenir l'énergie d'ancrage et la constante élastique $K_{13}$. Les prévisions de la théorie ont été vérifiées expérimentalement à l'interface nématique-isotrope du cristal liquide $7 \mathrm{CB}$. La géométrie et la dépendance en champ magnétique des déformations de la surface sont en accord satisfaisant avec les prédictions théoriques. On déduit des expériences une borne supérieure à la constante élastique $K_{13}$.
\end{abstract}

\begin{abstract}
Structural deformations of a nematic-isotropic interface induced by magnetic field have been predicted some years ago by de Gennes. In this paper we extend the de Gennes' theory in order to account for the finite anchoring energy of the director at the interface, for the anisotropy of the elastic constants $K_{11}$ and $K_{33}$ and for the effect of the elastic constant $K_{13}$ introduced by Nehring and Saupe. We obtain a simple analytical expression which relates the maximum tilt angle of the interface to the surface director polar angle, to the elastic constants, to the surface tension and to the magnetic field. Therefore a measurement of this tilt furnishes a direct method to measure the $K_{13}$ constant which cannot be measured by standard techniques. The predictions of the theory have been verified experimentally at the nematic-isotropic interface of the liquid crystal 4-cyano-4'-n-heptylbiphenyl (7CB). Both the geometry and the magnetic field-dependence of the surface deformations are found to agree satisfactorily with the theoretical predictions. An upper limit to the elastic constant $K_{13}$ is obtained from the experiment.
\end{abstract}

\section{Introduction.}

Nematic liquid crystals (L.C.) exhibit many typical defects (disclination lines or points) which can occur either in the bulk of the specimen or on the surfaces. Surface defects have been extensively investigated in the last years [1-7]. The characteristic feature of surface disclination lines is that they are wider than bulk disclination lines. This behaviour indicates that the average orientation of the molecular axes, defined by the unit vector $\mathbf{n}$ (director), changes in a continuous way across the disclination line. Figure 1 shows schematically the director-field near a wedge disclination line which lies along the $y$-axis at the interface of a nematic L.C. with a solid medium. Here we assume

$\left({ }^{+}\right)$Research supported in part by MPI and in part by CNR. that the easy polar angle of the director with the axis orthogonal to the interface is $+\theta_{\mathrm{t}}$ at the left of the disclination line and $-\theta_{\mathrm{t}}$ at the right, far away from the defect. Therefore, a distortion of the surface director-orientation has to occur close to the disclination line. The director-field $\mathbf{n}(\mathbf{r})$ can be obtained by minimizing the sum of the bulk Frank-Oseen elastic free energy [8] and of the surface anchoring energy of the director at the interface [9]. The characteristic length $s$ of the director distortion (see Fig. 1) corresponds to a compromise between the elastic contribution which is minimized for $s \rightarrow \infty$ and the anchoring contribution which is minimized for $s \rightarrow 0$. This length is of order of $K /\left(2 W_{0}\right)$ [4], where $K$ is an average elastic constant and $W_{0}$ is the anchoring energy coefficient [4].

A more complex behaviour can be expected at the interface between a nematic L.C. and an isotropic 


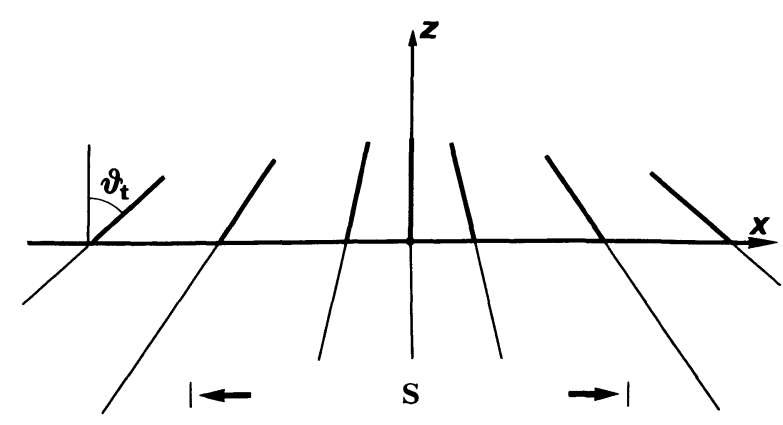

Fig. 1. - Schematic arrangement of the director near a wedge surface disclination line at the interface between a nematic L.C. and a solid medium. The disclination line is assumed to lie along the $y$-axis. $s$ is the characteristic length associated to director variations close to the disclination line whilst $\theta_{\mathrm{t}}$ is the easy polar angle of the director at the interface. Near the disclination line there is an excess of surface anchoring energy due to a tilt between the local director and the easy axis.

fluid. In this case the interface can deform itself in such a way to reduce the total free energy of the system as schematically shown in figure $2 \mathrm{a}$. In fact, a suitable tilt $\delta$ of the interface near the disclination line can reduce the angle between the director and the easy axis and thus, the anchoring energy. Therefore deformations of the interface can be energetically favoured.

A similar effect can occur when a horizontal magnetic field is applied to the system of figure $2 \mathrm{a}$. The surface distortion induced by the magnetic field alone (neglecting the original effect of the disclination line) is schematically shown in figure $2 b$. In this case a suitable tilt of the interface reduces the angle between the director and the magnetic field and thus, reduces the magnetic free energy. Note that the tilt of the interface due to the magnetic field (Fig. 2b) is opposite to that induced by the disclination line of figure $2 \mathrm{a}$. Therefore one expects that the resulting average tilt angle of the interface due to both these contributions (disclination line + magnetic field) changes its sign as the magnetic field exceeds some critical value.

Surface distortions of the nematic-isotropic interface induced by a magnetic field or by asymmetric orientational boundary conditions have been predicted by de Gennes [10] some years ago.

The main assumptions of the de Gennes theory were : i) isotropic elastic constants $\left(K_{i i}=K\right)$,ii) strong anchoring energy, iii) homeotropic director orientation at the interface $\left(\theta_{t}=0\right)$. Both surface disclination lines and surface defect points were predicted.

Characteristic textures of surface singular points have been effectively observed at the nematic-isotropic interface of some nematic L.C. layers [11, 12]. These singular points are due to hybrid boundary conditions for the director alignment at the interfaces of the layer. More recently Yokoyama et al. [13] observed periodic surface deformations induced by an electric field at the nematic-isotropic interface of a N.L.C. These latter textures cannot be explained in terms of

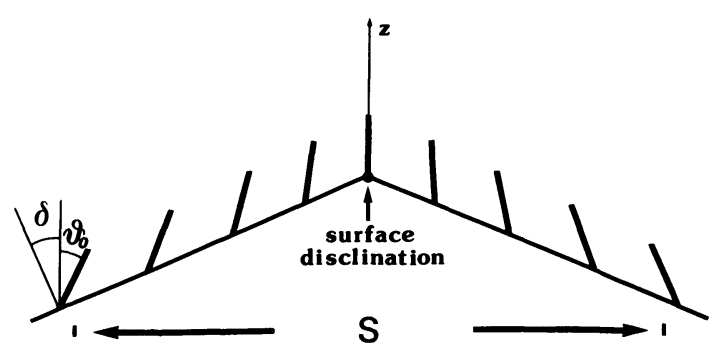

Fig. 2a. - Schematic arrangement of the director near a wedge surface disclination line at the interface between a nematic L.C. and an isotropic fluid medium. The tilt $\delta$ of the interface in the region close to the disclination line modifies the angle $\theta_{0}$ between the director and the axis orthogonal to the interface. Therefore a suitable sign of the $\delta$-angle can contribute to reduce the angle between the director and the easy axis at the interface and thus, the excess of anchoring energy. Surface tension and gravitational free energy are the restoring mechanisms which oppose to the tilt of the surface. Note that the physical mechanism responsible for surface deformations occurs in the small region where the director is not oriented along the easy axis $(X<3 s)$.

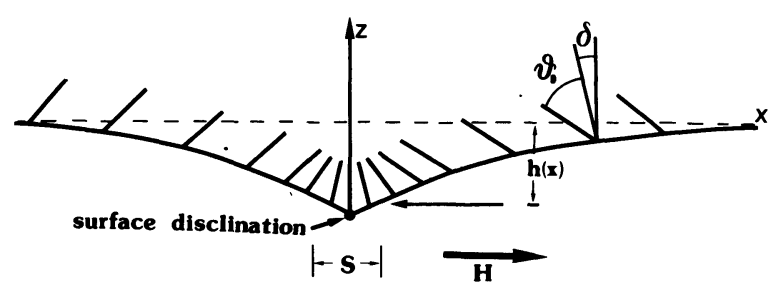

Fig. 2b. - Schematic view of the surface deformations induced by the magnetic field. At a large distance from the disclination line the director is nearly oriented along the easy axis (angle $\pm \theta_{\mathrm{t}}$ with respect to the axis orthogonal to the interface). A tilt $\delta$ of the interface of the kind shown in the figure reduces the polar angle between the director and the magnetic field and thus, the magnetic free energy. Note that this latter effect gives an important contribution also at large distances from the disclination line. In fact the characteristic length of the surface deformations is found to be some order of magnitude greater than the $s$ characteristic length. In general both the effects shown in figure $2 a$ and figure $2 b$ contribute to give the observed deformations of the interface.

the de Gennes theory. As a matter of fact the domains orientation is orthogonal with respect to that predicted by the de Gennes theory. At the best of our knowledge, no experimental observation of surface deformations induced by a magnetic field has been yet reported in the literature. Furthermore surface deformations have not been quantitatively investigated in the previous experiments [11-13].

In this paper we report the first experimental evidence of surface deformations induced by a magnetic field at the interface between isotropic and anisotropic phase of a nematic L.C. The geometry and the dependence on the magnetic field of these surface deformations are investigated in detail. 
In section 1 we extend the de Gennes theory in order to account for the effect of the anisotropy of the elastic constants $\left(K_{11} \neq K_{33}\right)$, of the tilted easy director orientation $\left(\theta_{t} \neq 0\right)$ and of the finite value of the anchoring energy. Furthermore we consider also the contribution of the elastic constant $K_{13}$ which appears in the generalized expression of the elastic free energy density proposed by Nehring and Saupe $[14,15]$. This elastic constant has been demonstrated to play an important role in the case of weak anchoring [16] since it influence the surface elastic torque. The main result of our theoretical analysis is a simple analytic expression for the local tilt angle of the interface. All the parameters (except $K_{13}$ ) which appear in this expression can be measured by different standard methods. Therefore a direct measurement of the tilt angle of the interface allows to obtain, in a way of principle, a direct measurement of the elastic constant $K_{13}$. This is a peculiar feature since all physical mechanisms which have been considered so far (Frederiks transition [17], hybrid cell threshold $[18], \ldots$ ) do not allow to obtain a direct unambiguous measurement of $K_{13}$. In fact, in all these cases the elastic constant $K_{13}$ appears in the boundary conditions together with another function : the anchoring energy $W$ [9], whose explicit analytic form is not known. This latter parameter cannot be measured by independent methods and thus, it is a hard matter to obtain from the experimental data both the elastic constant $K_{13}$ and the anchoring energy [16-18]. Approximate results can be obtained only by making some special conjectures about the dependence of the anchoring energy on the surface polar angle. Upper and lower limits for the elastic constant $K_{13}$ have been recently obtained by Barnik et al. [17] and by Barbero et al. [18] by studing the Frederiks transition and the hybrid cell transition, respectively, and by assuming different specific dependences of the anchoring energy on the surface director polar angle. Both these limits depend on the arbitrary assumptions made about the anchoring energy form.

Section 2 of this paper concerns the experimental investigation of the surface profile of the nematicisotropic interface of the nematic L.C. 4-cyano-4'n-heptylbiphenyl (7CB). By using a simple experimental method we have been able to evidence and investigate the quantitative features of surface deformations induced by a magnetic field. Our experimental results are in a satisfactory quantitative agreement with the predictions of the theory and allow to obtain an upper limit to the $K_{13}$ elastic constant.

\section{Theory of the surface deformations.}

Differential equations which describe the surface deformations and the corresponding director-field are obtained by minimizing the total free energy given by the sum of four contributions : the bulk elastic free energy, the bulk gravitational free energy, the surface anchoring energy and the surface tension. A great conceptual difference exists between the surface deformations induced by a disclination line (Fig. 2a) and those induced by the magnetic field (Fig. 2b). In the first case the physical interactions responsible for the tilt $\delta$ of the interface are confined to the narrow region $(s \sim 1 \mu \mathrm{m})$ close to the disclination line where the director polar angle differs appreciably from the easy value $\left( \pm \theta_{\mathrm{t}}\right)$. In fact is just in this region that a tilt of the interface can lower appreciably the surface anchoring energy. On the contrary the interactions due to the magnetic field occur everywhere at the interface (see caption of Fig. 2b). Therefore, in the first case, the theoretical analysis cannot neglect details of the distortion of the director-field near the disclination line. In particular the presence of a twist of the director near the disclination line must be accounted for. This fact makes very difficult the theoretical analysis and no analytical solution can be obtained in this case. On the contrary, the magnetic field effect can be investigated by neglecting the director distortion near the disclination line. This approximation is well justified since the characteristic length $\xi$ associated to director distortions near the $y z$ vertical plane of figure $2 b$ is much smaller than the characteristic length $\lambda$ of deformations of the interface. In the present experiment, for example, $\xi<20 \mu \mathrm{m}$ and $\lambda \sim 1 \mathrm{~mm}$. In the following we will get a detailed theoretical analysis of surface deformations induced by the magnetic field.

Consider the interface between an ordinary isotropic fluid and a nematic L.C. in the presence of a vertical gravity field and of an uniform magnetic field $H$ aligned along the horizontal $x$-axis. A wedge surface disclination line of the kind shown in figure 1 is assumed to lie along the horizontal $y$-axis. The nematic L.C. is in the bottom semispace $(z<0)$. The isotropic and anisotropic samples are assumed to be infinitely extended along the $z$-and $y$-axis. Far away from the disclination line the interface is parallel to the $x y$ plane. The bidimensional director-field in the $x z$ plane, is

$$
\mathbf{n} \equiv(\sin \theta(x, z), 0, \cos \theta(x, z))
$$

where $\theta(x, z)$ represents the polar angle which the director makes with the axis orthogonal to the interface. The total free energy of the system can be written in the form :

$$
\begin{array}{r}
\mathcal{F} \equiv \int \mathrm{d} x\left\{\left[\gamma\left(\theta_{0}, \delta\right)+W\left(\theta_{0}\right)+\gamma_{\mathrm{eq}}\right]\right. \\
\left(1+\frac{\delta^{2}}{2}\right)+ \\
\left.+\frac{\Delta e g h^{2}}{2}\right\}
\end{array}
$$

where $\theta_{0}=$ surface polar angle between the director and the axis orthogonal to the interface $\left(\theta_{0}<0\right.$ for $x>0$ and $\theta_{0}>0$ for $x<0$ ), $\gamma_{\mathrm{eq}}=$ equilibrium surface tension, $\Delta e=$ density difference between anisotropic and isotropic fluid, $g=$ gravity acceleration, $h(x)=$ local vertical displacement of the interface, 
$\delta=\mathrm{d} h / \mathrm{d} x, W\left(\theta_{0}\right)=$ anchoring energy and $\gamma\left(\theta_{0}, \delta\right)=$ excess of surface free energy due to the bulk elastic free energy and to the bulk magnetic free energy. The expression of $\gamma\left(\theta_{0}, \delta\right)$ (far away from the disclination line) is

$$
\begin{aligned}
& \gamma\left(\theta_{0}, \delta\right)=\int_{-\infty}^{h(x)} \mathrm{d} z^{\prime}\left\{\frac{1}{2} K_{33}\left(1+\eta \sin ^{2} \theta\right)\left(\frac{\mathrm{d} \theta}{\mathrm{d} z^{\prime}}\right)^{2}+\right. \\
& \left.+\frac{1}{2} \chi_{\alpha} H^{2} \cos ^{2}(\theta-\delta)\right\}-\left.K_{13} \frac{\sin 2 \theta_{0}}{2} \frac{\mathrm{d} \theta}{\mathrm{d} z^{\prime}}\right|_{z^{\prime}=h(x)}
\end{aligned}
$$

where $z^{\prime}$ represents a local coordinate orthogonal to the interface, $K_{\mathbf{3 3}}=$ bend elastic constant, $\eta=\left(K_{11}-K_{33}\right) / K_{33}=$ relative anisotropy of bend and splay elastic constants, $K_{13}=$ Nehring-Saupe elastic constant $[14,15]$ and $\chi_{\alpha}=\chi_{\|}-\chi_{\perp}=$ anisotropy of the diamagnetic susceptibility. In equation (3) we have neglected terms of order $\left(\frac{\partial \theta}{\partial x}\right)^{2} \ll\left(\frac{\partial \theta}{\partial z^{\prime}}\right)^{2}$. The contribution of the $K_{24}$ elastic constant vanishes in this geometry [18]. The bulk director orientation satisfies the equation :

$$
\frac{\partial \theta}{\partial z^{\prime}}= \pm \frac{\cos (\theta-\delta)}{\xi \sqrt{1+\eta \sin ^{2} \theta}}
$$

where $\xi=\sqrt{\frac{K_{33}}{\chi_{\alpha}}} \frac{1}{H}$ is the magnetic coherence length and the signs + and - stand for $x>0$ and $x<0$, respectively. By substituting equation (4) into equation (3) one gets (for $\eta<0$ ) :

$\gamma\left(\theta_{0}, \delta\right)=\frac{K_{33}}{2 \xi} \cos ^{2} \delta\left[\left(1+\eta \cos ^{2} \delta\right)^{1 / 2}+\frac{\arcsin \sqrt{-\eta} \cos \delta)}{\sqrt{-\eta} \cos \delta}\right]$

$$
\begin{aligned}
& +\frac{K_{33}}{2 \xi} \sin ^{2} \delta\left[\left(1+\eta \cos ^{2} \delta\right)^{1 / 2}+\frac{\operatorname{arcsinh}\left(\frac{\sqrt{-\eta} \sin \delta}{\sqrt{\eta+1}}\right)}{\sqrt{-\eta} \sin \delta}(1+\eta)\right] \\
& \pm \frac{K_{33}}{2 \xi} \sin \theta_{0} \cos \delta\left[\left(1+\eta \sin ^{2} \theta_{0}\right)^{1 / 2}+\frac{\arcsin \left(\sqrt{-\eta} \sin \theta_{0}\right)}{\sqrt{-\eta} \sin \theta_{0}}\right] \\
& \mp \frac{K_{33}}{2 \xi} \sin \delta \cos \theta_{0}\left[\left(1+\eta \sin ^{2} \theta_{0}\right)^{1 / 2}+\frac{(1+\eta) \operatorname{arcsinh}\left(\frac{\sqrt{-\eta} \cos \theta_{0}}{\sqrt{\eta+1}}\right)}{\sqrt{-\eta} \cos \theta_{0}}\right] \\
& \mp \frac{K_{13} \sin 2 \theta_{0} \cos \left(\theta_{0}-\delta\right)}{2 \xi\left(1+\eta \sin ^{2} \theta_{0}\right)^{1 / 2}} .
\end{aligned}
$$

The functions $\theta_{0}(x)$ and $h(x)$ can be obtained by minimizing the total free energy (Eq. (2)) with respect to $h(x)$ and $\theta_{0}(x)$. Therefore, we get the following Euler-Lagrange equations :

$$
\frac{\partial}{\partial \theta_{0}} \gamma\left(\theta_{0}, \delta\right)=-\frac{\partial}{\partial \theta_{0}} W\left(\theta_{0}\right)
$$

and

$$
-\frac{\partial}{\partial x}\left[\frac{\partial \gamma}{\partial \delta}\left(1+\frac{\delta^{2}}{2}\right)+\left(\gamma+W+\gamma_{\mathrm{eq}}\right) \delta\right]+\Delta e g h=0 .
$$

Equation (6) establishes the balance of surface torques whilst equation (7) furnishes the balance of surface vertical stresses responsible for surface deformations. Note that, in the limit case $\delta=\mathrm{d} h / \mathrm{d} x=0$ (rigid interface), equation (6) is the standard boundary condition for the director orientation [4]. If we look for solutions of equations (6) and (7) discontinuous at the disclination line $(x=0)$, the local derivative $\delta=\mathrm{d} h / \mathrm{d} x$ at this point is easily obtained by minimization of equation (2) and is given by (see also Refs. [9] and [10])

$$
\lim _{\substack{x \rightarrow 0^{+} \\ x \rightarrow 0^{-}}} \delta(x)= \pm \varepsilon=\frac{\left[-\frac{\partial}{\partial \delta} \gamma\left(\theta_{0}, \delta\right)\right]\left(1+\frac{\varepsilon^{2}}{2}\right)}{\gamma_{\mathrm{eq}}+\gamma\left(\theta_{0}, \pm \varepsilon\right)+W\left(\theta_{0}\right)} .
$$


Deformations of the interface are expected to be very small $(\mathrm{d} h / \mathrm{d} x \ll 1)$ and thus, one can look for the solution

$$
\theta_{0}(x)=\theta_{0}^{*}+\Delta \theta_{0}(x)
$$

where $\Delta \theta_{0}(x) \ll 1$ and $\theta_{0}^{*}$ is the solution of equation (5) for a rigid interface $(\delta=0)$. By linearizing equations (6) to (8) with respect to be small parameters $\delta$ and $\Delta \theta_{0}(x)$ we get :

$$
\begin{gathered}
\Delta \theta_{0}(x)=A\left(\theta_{0}^{*}\right) \delta \\
\frac{\mathrm{d}^{2} h}{\mathrm{~d} x^{2}}=\frac{h}{\lambda^{2}} \\
\varepsilon=\mp \frac{\left.\frac{\partial}{\partial \delta} \gamma\left(\theta_{0}, \delta\right)\right|_{\theta_{0}=\theta_{0}^{*}, \delta=0}}{\gamma_{\mathrm{eq}}+\gamma\left(\theta_{0}, \pm \varepsilon\right)+W\left(\theta_{0}\right)}
\end{gathered}
$$

where

$$
A\left(\theta_{0}^{*}\right)=\frac{-\left.\frac{\partial^{2}}{\partial \delta \partial \theta_{0}} \gamma\left(\theta_{0}, \delta\right)\right|_{\theta_{0}=\theta_{0}^{*}, \delta=0}}{\left.\frac{\partial^{2}}{\partial \theta_{0}^{2}}\left[\gamma\left(\theta_{0}, \delta\right)+W\left(\theta_{0}\right)\right]\right|_{\theta_{0}=\theta_{0}^{*}, \delta=0}}
$$

and

$$
\lambda=\sqrt{\frac{\gamma_{\text {eq }}+\gamma\left(\theta_{0}^{*}, 0\right)+W\left(\theta_{0}^{*}\right)+A\left(\theta_{0}^{*}\right) \frac{\partial^{2}}{\partial \theta_{0} \partial \delta} \gamma\left(\theta_{0}, \delta\right)+\frac{\partial^{2}}{\partial \delta^{2}} \gamma\left(\theta_{0}, \delta\right)}{\Delta e g}}
$$

Equation (11) admits the simple solution

$$
\frac{\mathrm{d} h}{\mathrm{~d} x}= \pm \varepsilon \mathrm{e}^{ \pm \frac{x}{\lambda}}
$$

where the signs - and + in the exponent stand for $x>0$ and $x<0$, respectively. The length $\lambda$ is the well known capillary length where the surface tension $\gamma_{\text {eq }}$ has been renormalized in order to account for the contributions of the anchoring energy and of the magnetic and elastic free energy. Notice that the contribution of these latter terms is negligible in many pratical cases. For example in the case of the nematicisotropic interface of 7CB [26] for $H=7 \mathrm{kG}$ we find $\gamma\left(\theta_{0}^{*}, \delta\right) \sim 2 \times 10^{-4} \mathrm{erg} / \mathrm{cm}^{2}$ and $W\left(\theta_{0}^{*}\right) \sim 0.4 \times$ $10^{-4} \mathrm{erg} / \mathrm{cm}^{2}$ which are much smaller than the interfacial surface tension $\gamma_{\mathrm{eq}}=1.8 \times 10^{-2} \mathrm{erg} / \mathrm{cm}^{2}$ [25]. This feature allows to greatly simplify equation (12) to equation (14) in many pratical cases. Note that in the case of strong anchoring and for $\theta_{t}=0, \eta=0$ and $K_{13}=0$ one recovers the original de Gennes results [10]. However, we point out that in the geometry considered by de Gennes the easy director polar angle at the interface was $\theta_{t}=0$ and the disclination was not present before turning on the magnetic field but it was generated by the m.f. itself. This latter feature implies that some energy has to be spent to generate the disclination line. Therefore the surface deformation appears only if the magnetic field exceeds a threshold value $H_{\mathrm{c}}$. On the contrary in our case $\theta_{\mathrm{t}} \neq 0$ and the disclination line already exists before turning on the m.f. and thus, no threshold field is expected. Furthermore in the de Gennes' case the surface director polar angle does not change with the magnetic field $(W \rightarrow \infty)$ and thus, the maximum tilt angle of the interface is found to be a linear function of the applied magnetic field. On the contrary, if the anchoring energy has a finite value, the surface polar angle is an increasing function of the m.f. and the maximum tilt angle shows a more complex dependence on $H$. Finally we remind that, in order to obtain equation (12), we have neglected the effect of the disclination line on the surface profile (see Fig. 2a). Therefore the effective maximum tilt angle of the surface will be :

$$
\varepsilon_{\text {eff }}=\varepsilon+\varepsilon_{\mathrm{D}}
$$

where $\varepsilon_{\mathrm{D}}$ corresponds to the original maximum tilt angle due to the disclination line in the absence of the m.f., whilst $\varepsilon$ is the maximum tilt angle induced by the m.f. (Eq. (12)). Equation (16) has been obtained in the hypothesis that the surface deformations due to the disclination line and to the magnetic field are completely decoupled due to the very different characteristic lengths associated to these two effects. This assump- 
tion is fully justified by our experimental results which show that the variation of the maximum tilt angle induced by the m.f. does not depend on the value of this angle at a zero m.f.

In most of the experimental works the m.f. is not able to modify greatly the surface director polar angle $\left(\theta_{0} \sim \theta_{t}\right)$ and thus, one can use the following approximate form of the anchoring energy :

$$
W=W_{0}\left(\theta_{0} \pm \theta_{t}\right)^{2}
$$

where $W_{0}$ is the anchoring energy coefficient. By substituting the explicit forms of $\gamma\left(\theta_{0}\right)$ (Eq. (5)) and $W\left(\theta_{0}\right)($ Eq. (17)) in equations (6)-(12) we obtain two analytical equations for $\varepsilon$ and $\theta_{0}$. The coefficients which appear in these equations are $K_{33}, \eta, K_{13}, \chi_{\alpha}, \Delta e, \gamma_{\mathrm{eq}}$ and $W_{0} . K_{33}, \eta, \chi_{\alpha}, \Delta e$ and $\gamma_{\text {eq }}$ can be measured by standard methods [19-22] and thus, a measurement of the surface director polar angle $\theta_{0}$ and of the maximum tilt angle $\varepsilon$ of the interface, allows to measure directly both the anchoring energy coefficient and the $K_{13}$ elastic constant. This is, at the best of our knowledge, the first unambiguous method to measure $K_{13}$.

Before discussing our experimental results we want to emphasize this latter point. Consider, for instance, the special case of a rigid interface $(\delta=0)$. If $\theta_{0}-\theta_{\mathrm{t}} \ll 1$ the boundary condition for the surface polar angle (Eq. (6)) becomes :

$$
\pm \frac{K_{33}}{\xi} \cos \theta_{0} \sqrt{1+\eta \sin ^{2} \theta_{0}}\left\{1-\frac{K_{13}}{K_{33}}\left[\frac{\cos ^{2} \theta_{0}-2 \sin ^{2} \theta_{0}-2 \eta \sin ^{4} \theta_{0}}{\left(1+\eta \sin ^{2} \theta_{0}\right)^{2}}\right]\right\}=-2 W_{0}\left(\theta_{0} \pm \theta_{t}\right)
$$

Two unknown coefficients $\left(K_{13}\right.$ and $\left.W_{0}\right)$ appear in this boundary condition. Therefore, a measurement of the surface director polar angle $\theta_{0}$ versus the magnetic field does not allow to obtain these coefficients. Usually one bypasses this difficulty by assuming $K_{13}=0$, in order to obtain $W_{0}$ from equation (18). However the anchoring energy coefficient measured in this way does not correspond, in principle, to the true anchoring energy coefficient. In an analogous way, for $\left|\theta_{0}\right|-\left|\theta_{t}\right| \sim 1$, equation (6) does not allow to obtain the elastic constant $K_{13}$ since the theoretical dependence of $W$ on $\theta_{0}$ is not known. The same kind of difficulty concerns, for example, the case of the Frederiks transition in a nematic layer submitted to a magnetic field [17] or the case of a nematic layer between two rigid surfaces giving different easy director orientations (hybrid cell [18]). On the contrary, in the case of a deformable interface, equation (6) and equation (12) furnish two independent equations for $\theta_{0}$ and $\delta$ which allow to measure both $K_{13}$ and $W\left(\theta_{0}\right)$. Furthermore the measurement of the $x$-dependence of the local tilt angle of the interface allows to measure the interfacial surface tension (Eq. (14)).

\section{Experiment.}

In this section we report the first experimental evidence of surface deformations induced by a magnetic field at a nematic-isotropic interface. The geometry and the magnetic field dependence of these structural deformations are investigated in detail. A satisfactory agreement with the theoretical predictions is found.

The experimental apparatus is shown in figure 3. The nematic sample used for the experiment is 4-cyano-4'-n-heptylbiphenyl (7CB) produced by BDH. This nematic material exhibits a high chemical stability [23] that ensures a good reproducibility of the



Fig. 3. - Experimental apparatus used to measure the local tilt of the interface. A He-Ne laser beam is focalized on the nematic-isotropic interface by means of the lens $L$. The reflected beam passes throught the beam splitter $G$ and impinges on the screen S. A horizontal magnetic field is applied parallel to the interface. The incidence point can be changed in a continuous way by means of a $x-y$ translation stage.

experimental results. The clearing temperature of this nematic L.C. is $T_{\mathrm{c}}=42.8^{\circ} \mathrm{C}$. The specimen is enclosed in a cylindrical glass cell $2-\mathrm{cm}$ high and $1.5-\mathrm{cm}$ large having two plane glass windows on top and bottom, respectively. These windows are in thermal contact with two copper plates maintained at two different temperatures $T_{1}$ and $T_{2}$ which are held fixed within $\pm 0.05^{\circ} \mathrm{C}$. The upper copper plate has a $1-\mathrm{cm}$ diameter hole which allows a laser beam to pass through. The temperature of the top plate is higher than the clearing temperature $T_{\mathrm{c}}$, whilst the temperature of the bottom plate is lower than $T_{\mathrm{c}}$. Under these conditions a nematic-isotropic interface occurs at a given height of the sample. By changing the temperatures $T_{1}$ and $T_{2}$ one can modify either the position of the nematic-isotropic interface or 
the applied temperature gradient. The typical value of the applied temperature gradient is $5 \% / \mathrm{cm}$. A great care is devoted to obtain a temperature gradient parallel to the gravity field in order to avoid mechanical instabilities of the interface. The cylindrical glass cell lies between the two polar expansions of an electromagnet which gives magnetic fields up to $7.6 \mathrm{kG}$.

The experimental method to measure the local tilt angle of the interface is shown in figure 3. A $10 \mathrm{~mW} \mathrm{He}-\mathrm{Ne}$ laser beam is focalized on the interface by a $30-\mathrm{cm}$ focal length optical lens in such a way to obtain a beam waist (minimum diameter of the beam) of about $150 \mu \mathrm{m}$. Under these conditions the angular divergence of the reflected beam is $\sim 0.006 \mathrm{rad}$. The reflection angle of the laser beam is determined by measuring the displacement of the laser spot on a white screen at a distance of $125 \mathrm{~cm}$ from the interface. The reflection angle $\alpha$ is related to the local gradient $\nabla h(x, y)$ by the simple expression :

$$
\alpha=2|\nabla h(x, y)| .
$$

The cylindrical cell, containing the nematic L.C., is placed on a micrometric translation stage that allows to shift the interface along two orthogonal axes $x$ and $y$ in the horizontal plane. In this way we can make a detailed analysis of space variations of $\nabla h(x, y)$.

Before discussing our experimental results we address briefly some important issues. The theoretical analysis performed in the previous section concerned the minimization of the Helmholtz free energy which is defined for a physical system at the thermal equilibrium. On the contrary, the physical system we investigate here is a near-equilibrium system since the temperature is not uniform. However the temperature gradient is small and thus, the characteristic length scale of temperature variations is much greater than typical intermolecular distances. Therefore is yet possible to define a local temperature $T(x, y, z)$ and a local Helmholtz free energy $F(x, y, z)$. The non-uniformity of the temperature has two consequences : 1) the physical coefficients $\left(K_{33}, K_{13}, \ldots\right)$ which occur in the equations of the previous section are temperature-dependent ; 2) thermohydrodynamic instabilities can occur if the temperature gradient exceeds some threshold value [24]. The first point can be neglected in our problem since the thickness of the distorted layer close to the interface is of order of a few magnetic coherence lengths $\xi(\xi<20 \mu \mathrm{m})$. Therefore the maximum temperature variation across the distorted layer is $\Delta T<3 \frac{\partial T}{\partial z} \xi \lesssim 3 \times 10^{-2}{ }^{\circ} \mathrm{C}$.

In order to exclude the influence of thermohydrodynamic instabilities in our experiment, we have changed the temperature gradient in the range from $1^{\circ} \mathrm{C} / \mathrm{cm}$ to $10^{\circ} \mathrm{C} / \mathrm{cm}$ without observing any important variation of the surface profile. This ensures that thermohydrodynamic instabilities do not affect our experiment. Furthermore we have also verified that the main features of surface distortions did not change when the temperatures $T_{1}$ and $T_{2}$ of the two copper plates were modified in such a way to shift the vertical position of the n-i-interface without changing the temperature gradient.

A continuous translation of the nematic cell in the horizontal $x y$ plane allows us to obtain the topography of the nematic-isotropic interface. If we look at the spot of the reflected laser beam on the white screen we note that it shifts continuously as the nematic cell is translated. This feature indicates that the interface is not plane. In the correspondence of some particular regions of the interface one can observe a sharp variation of the reflected beam : the reflected laser beam splits into two symmetric components. This means that a surface defect of the same kind of those schematically shown in figure $2 a$ and figure $2 b$ is present at the interface. An accurate analysis of the reflected beam shows that the observed defects correspond to curved lines on the interface. Typically some defects can be observed on the same interface having an average distance the one from the other of a few millimeter. The spatial arrangement of these defects is not regular. Figure 4 shows schematically the typical surface topography along the $x$ axis. The most reproducible data on the magnetic field dependence of these textures are obtained when we look at regions of the interface where the local gradient $(\nabla h)$ in the absence of the m.f. lies in the $x-z$ vertical plane. This implies that, in the proximity of the incidence region, the disclination line is oriented along the $y$-axis as assumed in the previous theoretical analysis.

The local tilt angle $\delta$ of the interface versus the $x$ distance from the disclination line is shown in figure 5. These results have been obtained in the case of a $5 \mathrm{kG}$ magnetic field. The slight asymmetry and the deviations from the exponential dependence are due to the presence of other disclination lines on the interface. In fact the average distance between disclination lines is a few millimeter, which is comparable with the capillary length $\lambda \sim 1 \mathrm{~mm}$. Another reason for these distortions can be also due to meniscus effects related to the finite size of the cylindrical glass cell which contains the nematic L.C. The capillary lengths measured in the correspondence of different disclination lines have the same value

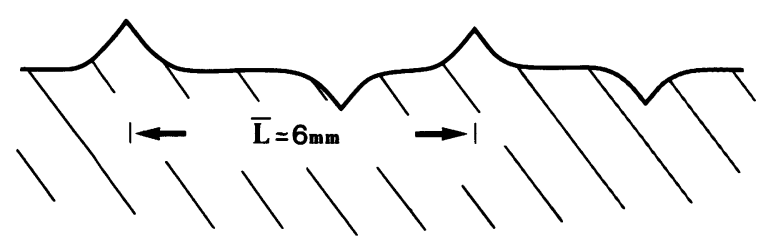

Fig. 4. - Schematic cross section of the sample in the $x z$ vertical plane. A sequence of hills and valleys occurs with an average distance of about $3 \mathrm{~mm}$. 


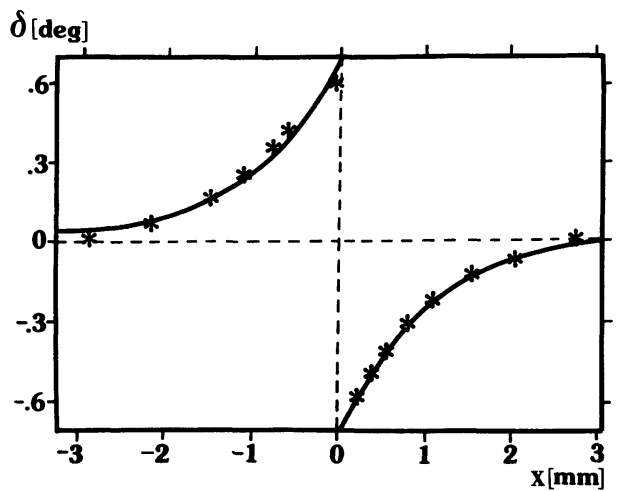

Fig. 5. - $x$-dependence of the local tilt angle $\delta$ of the nematic-isotropic interface when a magnetic field $H=5 \mathrm{kG}$ is applied. $x=0$ corresponds to the position of the singular line. The surface angle shows an abrupt variation in the correspondence of $x=0$. The full line corresponds to a $x$-dependence as $\delta=\mp A \mathrm{e}^{\mp x / \lambda}+B x+C$ with $A=0.7 \mathrm{deg}$., $B=-0.007 \mathrm{deg} . / \mathrm{mm}, C=0.02$ deg., and $\lambda=1.15 \mathrm{~mm}$. The $B$ and $C$ parameters account for spurious effects due to the presence of other disclination lines on the interface.

within $20 \%$. By comparing the experimental results with equations (15) and (14) one can obtain the value of the $\lambda$-characteristic length

$$
\lambda=1.15 \pm 0.2 \mathrm{~mm} .
$$

This value corresponds to the average value of the capillary length measured on ten different disclination lines. By substituting this value together with $\Delta e=$ $2.1 \pm 0.3 \times 10^{-3} \mathrm{~g} / \mathrm{cm}^{3}$ in equation (14) and by neglecting the small contributions of $\gamma\left(\theta_{0}^{*}\right)$ and $W\left(\theta_{0}^{*}\right)$ we obtain $\gamma_{\text {eq }}=2.8 \pm 1.2 \times 10^{-2} \mathrm{erg} / \mathrm{cm}^{2}$. The capillary length $\lambda$ at the nematic-isotropic interface of 7CB has been already measured by us by using the sessile drop method in order to obtain the interfacial surface tension [25]. The measured value was $\lambda=$ $0.95 \pm 0.1 \mathrm{~mm}$ which corresponded to the surface tension $\gamma_{\mathrm{eq}}=1.8 \pm 0.7 \times 10^{-2} \mathrm{erg} / \mathrm{cm}^{2}$. From the experimental data of figure 5 we can obtain the maximum vertical displacement of the interface close to the disclination line for $H=5 \mathrm{kG}$ which is $h_{\max } \sim$ $(\mathrm{d} h / \mathrm{d} x)_{\max } \lambda \sim 20 \mu \mathrm{m}$.

Figure 6 shows the maximum tilt angle $\varepsilon_{\text {eff }}$ of the interface at $x=0$ versus the magnetic field intensity $H$. $\varepsilon_{\text {eff }}$ is not a linear function of the magnetic field. This feature is due to the finite value of the anchoring energy which allows the surface director orientation to change as the m.f. is increased. Note that the tilt angle of the interface does not vanishes when $H=0$. This residual tilt angle is due to the effect of the disclination line on the interfacial profile as already discussed in the Introduction (see Fig. 2a). The value of this angle is not the same for all disclination lines but can range from a minimum value $\sim-0.5^{\circ}$ to a maximum value $\sim-0.2^{\circ}$. On the contrary the effect of the magnetic field is well reproducible within our experimental accuracy $(10 \%)$. In parti-

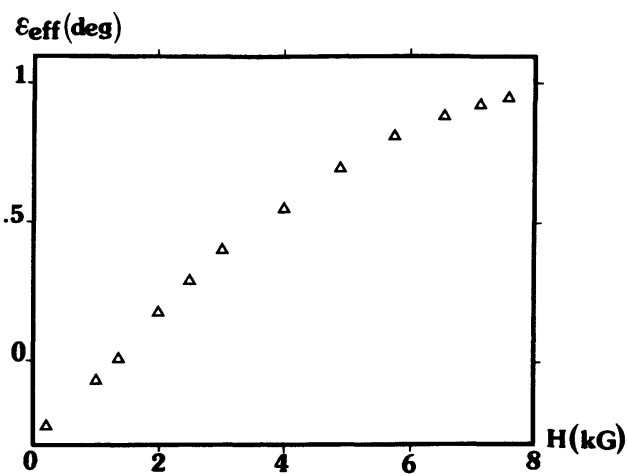

Fig. 6. - Maximum tilt angle of the interface versus the magnetic field. Note that there is an appreciable tilt of the interface also when $H=0$. This tilt is due to the effect of the disclination line alone as shown in figure $2 \mathrm{a}$ and it is not the same for all disclination lines. When $H=1.3 \mathrm{kG}$ the maximum tilt of the interface is zero. The non linear dependence of $\varepsilon_{\text {eff }}$ on the magnetic field is a consequence of the finite anchoring energy which allows the director to tilt with respect to the easy axis as the magnetic field is increased (Eq. (18) and Eq. (12)).

cular the difference $\varepsilon(H)=\varepsilon_{\text {eff }}(H)-\varepsilon_{\text {eff }}(0)$ is a well defined function which does not depend on the value $\varepsilon_{\text {eff }}(0)$. This observation strongly supports our previous conjecture of assuming the effects of the disclination line and of the m.f. as completely decoupled (Eq. (16)). According to the predictions of the previous section, the effect of the magnetic field on the surface profile is always opposite to that of the disclination line alone (see Fig. 2a and Fig. 2b). Therefore we always find a characteristic magnetic field $(0.7-1.5 \mathrm{kG})$ in the correspondence of which these two contributions are balanced and the interface becomes nearly plane. The value of this characteristic field depends on the zero-field angle $\varepsilon_{\text {eff }}(0)$. In the case of figure 6 the characteristic m.f. is $1.3 \mathrm{kG}$. In order to obtain the anchoring energy coefficient $W_{0}$ and the elastic constant $K_{13}$ from this experimental measurement one must known the dependence of the surface director polar angle $\theta_{0}$ on the m.f. This measure has been given by us in a recent paper [26]. The experimental method consisted in measuring the reflectivity coefficients for a laser beam which impinged at a normal incidence on the n-i-interface of 7CB. These reflectivity coefficients are related to the surface director polar angle and to the thickness of the interface. Therefore the measurement of both the reflectivity coefficients allows to obtain the surface director polar angle $\theta_{0}$. In reference [26] a great care was devoted to measure $\theta_{0}$ far away from the disclination line where the interface is almost plane. Therefore the here measured value of the surface polar angle corresponds to the $\theta_{0}^{*}$-angle defined in equation (9). Our experimental results indicate that $\theta_{0}^{*}$ is an almost linear function of the magnetic field which has the values $\theta_{0}^{*}=52.6 \pm 6^{\circ}$ for $H=0$ and $\theta_{0}^{*}=65.5 \pm 6^{\circ}$ for $H=7.6 \mathrm{kG}$. The 
uncertainty on the difference $\Delta \theta_{0}^{*}=\theta_{0}^{*}(7.6)-\theta_{0}^{*}(0)$ is of order of $2^{\circ}$ which is smaller than the absolute uncertainty of the single measurements.

Figure 7 shows the dependence of $\varepsilon=\varepsilon_{\text {eff }}(H)$ $\varepsilon_{\text {eff }}(0)$ on the product $H \cos \theta_{0}(H)$, where $\theta_{0}$ is the polar angle near $x=0$. This angle can be obtained from the experimental value $\theta_{0}^{*}$ measured in reference [26] by using equations (9), (10), (13) and (14). The dependence of $\varepsilon$ on $\cos \theta_{0} . H$ is expected to be linear if $K_{13}=0$ and $\eta=0$ (see Eqs. (12) and (5)). The full line in figure 7 corresponds to the theoretical behaviour expected for $K_{13}=0, K_{33}=2.2 \times$ $10^{-7} \mathrm{erg} / \mathrm{cm} \quad[27], \quad \eta=-0.1 \quad[27], \quad W_{0}=8 \times$ $10^{-4} \mathrm{erg} /\left(\mathrm{cm}^{2} \mathrm{rad}^{2}\right), \chi_{\alpha}=0.62 \times 10^{-7}$ e.s.u. [21] and $\gamma_{\text {eq }}=1.65 \times 10^{-2} \mathrm{erg} / \mathrm{cm}^{2}$. The value of the anchoring energy coefficient has been calculated by substituting the experimental values of $\theta_{0}^{*}(H)$ in equation (18) and by using the previous values of the elastic constants. This value is almost two times lower than that already given in reference [26] since this latter had been obtained by using the elastic constants given in reference [19] which have been demonstrated to be uncorrect. The correct elastic constants are given in reference [27]. The surface tension value which corresponds to the full line in figure 7 agrees satisfactorily with that of reference [25] $\left(\gamma_{\mathrm{eq}}=1.8 \pm\right.$ $\left.0.7 \times 10^{-2} \mathrm{erg} / \mathrm{cm}^{2}\right)$. Therefore, within the accuracy of the present experiment, our results are compatible with a zero value of the $K_{13}$ elastic constant. By accounting for the uncertainty of the coefficients $K_{33}(10 \%), \chi_{\alpha}(10 \%), \gamma_{\text {eq }}(40 \%)$ and of the surface director polar angle $(10 \%)$ and of the tilt angle of the interface $(10 \%)$ we get an upper limit for the Nehring-Saupe elastic constant :

$$
\left|K_{13}\right|<1.5 \times 10^{-7} \mathrm{erg} / \mathrm{cm} \sim 0.7 K_{33} .
$$

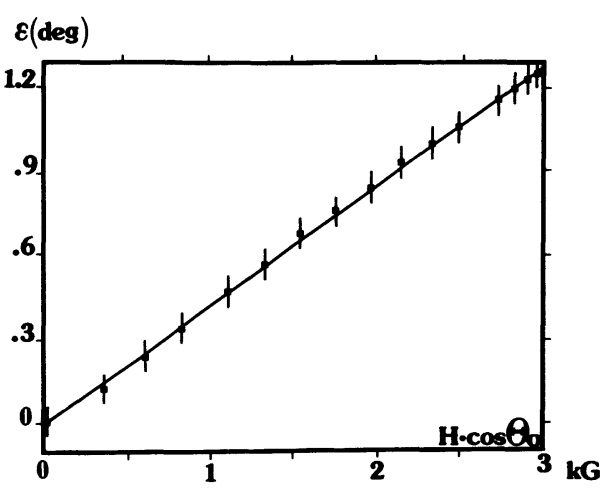

Fig. 7. - Magnetic field contribution to the maximum tilt angle of the interface $\left(\varepsilon=\varepsilon_{\text {eff }}(H)-\varepsilon_{\text {eff }}(H=0)\right)$ versus $H \cos \left(\theta_{0}\right)$, where $\theta_{0}$ is the surface director polar angle close to the disclination line. This angle can be calculated by substituting the measured polar angle $\theta_{0}^{*}[26]$ in equations (9), (10), (13) and (17). The full line corresponds to the theoretical behaviour expected for $K_{13}=0, K_{33}=$ $2.2 \times 10^{-7} \mathrm{erg} / \mathrm{cm} \quad[27], \quad \eta=-0.1 \quad[27], \quad \chi=0.62 \times$ $10^{-7}$ e.s.u. [21], $W_{0}=8 \times 10^{-4} \mathrm{erg} /\left(\mathrm{cm}^{2} \mathrm{rad}^{2}\right)$ and $\gamma_{\text {eq }}=1.65 \times 10^{-2} \mathrm{erg} / \mathrm{cm}^{2}$.
This upper limit is somewhat large as a consequence of the great uncertainty on the interfacial surface tension of the nematic-isotropic interface. Note that microscopic molecular models predict $K_{13}<0[14,15]$. Furthermore we note that our limit for $K_{13}$ is consistent with the previous upper and lower limits obtained by Barnik et al. $\left(0>K_{13}>-K_{33} / 2=\right.$ - $1.1 \mathrm{erg} / \mathrm{cm}[17])$ and by Barbero and Strigazzi $\left(K_{13}>-K_{33} / 2\right.$ [18]), who studied the behaviour of a nematic L.C. in an electric field or the behaviour of a hybrid nematic cell, respectively. However we point out that, in our case, no special assumption about the explicit dependence of the anchoring energy on the surface director polar angle has been made.

\section{Concluding remarks.}

In this paper we have extended the de Gennes' theory of surface deformations at the nematic-isotropic interface by accounting for the effects of the tilted easy director orientation $\left(\theta_{t} \neq 0\right)$, of the finite anchoring energy, of the anisotropy of the FrankOseen elastic constants and of the Nehring-Saupe elastic constant $K_{13}$. We shows that the measurement of the surface director polar angle and of the maximum tilt angle $\varepsilon$ of the interface furnishes, in principle, a direct and unambiguous method to measure both the anchoring energy coefficient and the $K_{13}$ elastic constant. This result cannot be obtained by standard methods which concern rigid interfaces. Furthermore a measurement of the capillary length $\lambda$ associated to surface deformations allows to obtain the surface tension of the nematic-isotropic interface. Therefore the analysis of surface deformations seems to be a promizing method to investigate interfacial properties of liquid crystals. However we remark that this latter technique can be used only in the case of interfaces between two fluid media having a little density difference and a small interfacial surface tension. This is just the case of the interface between isotropic and anisotropic phase of a nematic L.C.

In the second section of this paper we report the first quantitative investigation of surface defects generated by a magnetic field at the nematic-isotropic interface of the nematic L.C. 7CB. The qualitative and quantitative features of these defects agree satisfactorily with the predictions of the theory. Our experimental data are consistent with a zero value of the $K_{13}$ elastic constant and with a finite value of the anchoring energy coefficient given by $W_{0}=8 \pm 2 \times 10^{-4} \mathrm{erg} /\left(\mathrm{cm}^{2} \mathrm{rad}^{2}\right)$. The experimental results allow to obtain an upper limit to the $K_{13}$ elastic constant. Unfortunately the physical parameters of the nematic-isotropic interface are known with a poor accuracy and thus, also our experimental results are affected by a large uncertainty. We think that this technique should fournish more accurate results if applied to simpler systems as the interface between a conventional isotropic fluid and 
an anisotropic N.L.C. having comparable mass densities and a low interfacial surface tension. In this case, in fact, the physical parameters of the interface $\left(\gamma_{\mathrm{eq}}, \Delta e, \ldots\right)$ could be measured with a much greater accuracy, since this is an equilibrium system. Furthermore, measurements of the temperature-dependence of surface parameters should be also possible in this case.

\section{References}

[1] Williams, C., Vitek, V. and Kleman, M., Solid State Commun. 12 (1973) 581.

[2] Kleman, M. and Williams, C. E., Philos. Mag. 28 (1973) 725.

[3] Meyer, R. B., Solid State Commun. 12 (1973) 585.

[4] Vitek, V. and Kleman, M., J. Physique 36 (1975) 59.

[5] Ryschenkow, G. and Kleman, M., J. Chem. Phys. 64 (1976) 1.

[6] Raganath, G. S., J. Physique Colloq. 40 (1979) C3-87.

[7] Melzer, D., Van Es., A., Nabarro, F. R. N. and Godinho, E., Philos. Mag. 44A (1981) 835.

[8] Oseen, C. W., Trans. Faraday Soc. 29 (1933) 883.

Frank, F. C., Discuss. Faraday Soc. 25 (1958) 19.

[9] DE Gennes, P. G., Introduction to the Physics of Liquid Crystals (Oxford : The Clarendon Press) 1979.

[10] De Gennes, P. G., Solid State Commun. 8 (1970) 213.

[11] Meyer, R. B., Mol. Cryst. Liq. Cryst. 16 (1972) 355.

[12] Madhusudana, N. V. and Sumathy, K. R., Mol. Cryst. Liq. Cryst. 92 (1983) 193.

[13] Yokoyama, H., Kobayashi, S. and Kamei, H., Deformations of a planar nematic-isotropic interface in uniform and nonuniform electric fields, paper presented at the $\mathbf{X}$ International Conference on Liquid Crystals (York).

[14] Nehring, J. and Saupe, A., J. Chem. Phys. 54 (1971) 337.

[15] Nehring, J. and Saupe, A., J. Chem. Phys. 56 (1972) 5527.

[16] Derzhanski, A. I. and Hinov, H. P., Phys. Lett. 56A (1976) 465.

Hinov, H. P., J. Physique Lett. 38 (1977) L-215.
Derzhanski, A. I. and Hinov, H. P., J. Physique 38 (1977) 1013.

Hinov, H. P. and Derzhanski, A. I., J. Physique Colloque 40 (1979) C3-305.

Derzhanski, A. I., Petrov, A. G. and Mitov, M. D., J. Physique 39 (1978) 273.

[17] Barnik, M. I., Blinov, L. M., Korkishko, T. V., UMANSKy, B. A. and Chigrinov, V. G., Mol. Cryst. Liq. Cryst. 99 (1983) 53.

[18] Barbero, G. and Strigazzi, A., Influence of the surface-like volume elasticity on the critical thickness of a hybrid aligned nematic cell, to be published on J. Physique.

[19] Karat, P. P. and Madhusudana, N. V., Mol. Cryst. Liq. Cryst. 40 (1977) 239.

[20] Sherrel, P. L. and Crellin, D. A., J. Physique Colloq. 40 (1979) C3-211.

[21] Dunmur, D. A. and Miller, V. H., J. Physique Colloq. 40 (1979) C3-141.

[22] Williams, R., Mol. Cryst. Liq. Cryst. 35 (1976) 349.

[23] Gray, G. W., J. Physique Colloq. 36 (1975) C1-337.

[24] Dubois-Violette, E., C. R. Hebd. Séan. Acad. Sci. B-273 (1971) 923.

[25] Faetti, S. and Palleschi, V., Measurement of the interfacial tension between nematic and isotropic phase of some cyanobiphenyls, J. Chem. Phys. (in press).

[26] Faetti, S. and Palleschi, V., J. Physique Lett. 45 (1984) L-313.

[27] Madhusudana, N. V. and Pratibha, R., Mol. Cryst. Liq. Cryst. 89 (1982) 249. 